\title{
Fatigue of Friction Stir Welded 2024-T351 Aluminium Alloy
}

\author{
D. Booth and I. Sinclair \\ School of Engineering Sciences, University Of Southampton, Southampton, SO17 1BJ, UK
}

Keywords: Friction Stir Welding, fatigue, 2024, initiation, propagation

\begin{abstract}
Fatigue failure characteristics of friction stir welds in $13 \mathrm{~mm}$ gauge 2024-T351 plate have been assessed. Failure occurred from either the weld region (nugget/flow arm) or from the material immediately surrounding the weld. Fatigue failure from the surrounding material was essentially conventional, initiating from large S-phase intermetallic particles and growing in a macroscopic mode I manner. Corresponding fatigue lives were seen to be comparable to parent plate and results previously reported for similar welds in thinner plate. Failure over the weld region was identified with discontinuities in the macroscopic flow pattern of the weld flow arm. Subsequent crack growth showed pronounced macroscopic crack deflection around the 'onion ring' structure of the weld nugget. The bands making up the onion rings were identified with variations in local hardness levels, consistent with a mechanical contribution to the crack deflection process.
\end{abstract}

\section{Introduction}

Significant interest has been shown in the use of advanced welding techniques for aircraft structures, particularly given the design and manufacturing benefits they afford over established mechanical joining methods [1]. Whilst a variety of welding methods have been identified for airframe structures, friction stir welding (FSW) is an important candidate technique that is distinctive in being a low energy, solid-state process [2].

A key factor in the exploitation of FSW techniques in primary airframe structures will of course be the associated fatigue characteristics. It is significant to note that various aspects of established airframe design are closely linked to the intrinsic interactions between cracks and mechanically fastened components (such as the incidence of crack initiation at rivet holes and the inherent damage tolerance associated with the multiple load paths in riveted structures). The extensive use of welded airframe components (via FSW techniques or otherwise) therefore represents an important shift in the treatment of damage tolerance in airframes, requiring an understanding of cracks initiation characteristics and corresponding implications for crack growth.

\section{Materials}

Microstructure. Single-pass friction stir welded panels of $13 \mathrm{~mm}$ gauge 2024-T351 plate were provided by Airbus UK (Filton) in the as-welded condition, with the weld line running parallel to the longitudinal (rolling) direction of the parent plate. The overall weld structure is illustrated in Fig. 1. A well-defined central nugget may be seen, exhibiting a distinct annular banded (or 'onion ring') structure in keeping with various reports within the literature [2,3]. It is also possible to identify a 'flow arm' region in between the nugget and the surface on which welding was carried out. 


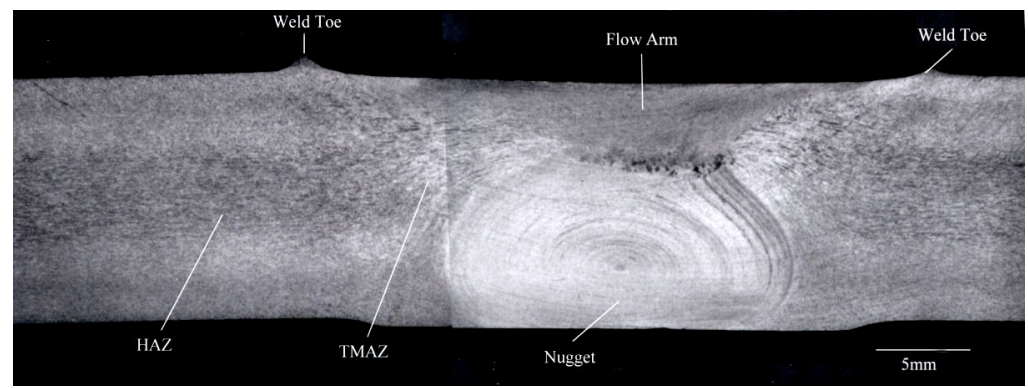

Figure 1 Optical macrograph of friction stir welded $13 \mathrm{~mm}$ gauge 2024-T351

Further out from the weld line the thermomechanically-affected zone (TMAZ) and heat-affected zone (HAZ) may be identified on each side of the weld. The characteristic deformation of the parent plate grain structure associated with the TMAZ is particularly illustrated in Fig. 2 for the retreating side of the weld (i.e. the side of the weld where the direction of probe rotation and the work piece traverse direction are opposite). In keeping with various reports in the literature, a fine, equiaxed grain structure was identified within the weld nugget and flow arm regions (grains up to $\sim 5 \mu \mathrm{m}$ diameter). The intermetallic particle distribution of the weld region was also seen to be refined in relation to the parent plate, with maximum particle dimensions of the order of $1-2 \mu \mathrm{m}$ in the nugget and flow arm (as opposed to particles of up to $10 \mu \mathrm{m}$ in the parent microstructure).

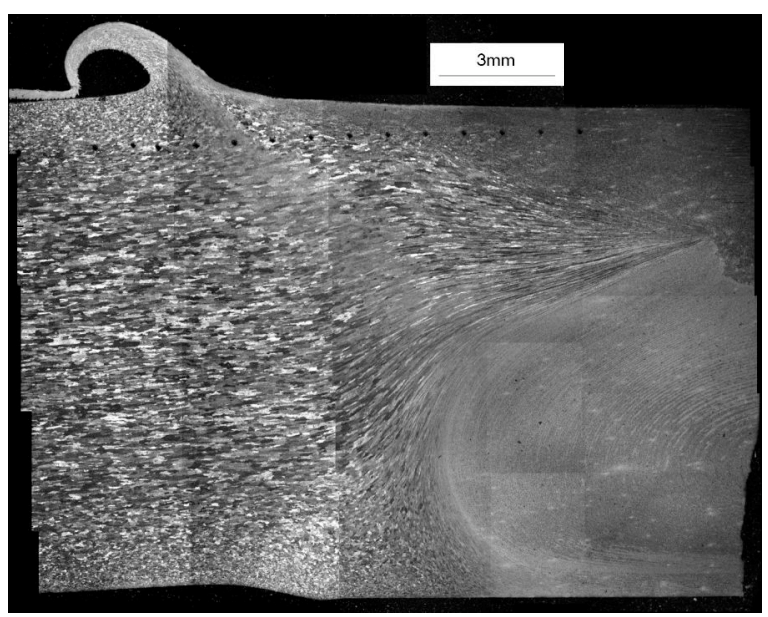

Figure 2 Optical micrograph of the retreating side of friction stir weld in $13 \mathrm{~mm}$ gauge 2024-T351

Hardness Characteristics. Fig. 3(a) shows an optical macrograph of the overall weld structure, along with Fig. 3(b), which is a 2D hardness map of the same region at the same scale. The general outline of the weld is delineated in the hardness map, with both the weld nugget and flow arm regions being associated with a central plateau in hardness levels. The line traces shown in Fig. 3(c) correspond to individual hardness profiles across the weld nugget (near its centre) and across the flow arm (approx. $2 \mathrm{~mm}$ below the parent plate surface). Going from the parent plate towards the weld line, both traces exhibit a small initial rise from the parent plate hardness level, which is followed by a gradual decline in hardness to a minimum either side of the nugget/flow arm. There is then a steep rise to a central plateau of intermediate hardness. The hardness trace through the weld nugget (identified as $4 \mathrm{~mm}$ from the weld back in Fig. 3(c)) shows a small but distinct dip in the central plateau region that is also evident in the weld nugget region of the map in Fig. 3(b). The plateau region in the weld flow arm (identified as $11 \mathrm{~mm}$ from the weld back in Fig. 3(b) and (c)) does not show a central dip and is significantly wider that both the nugget and the fine grained region of the flow arm itself. 


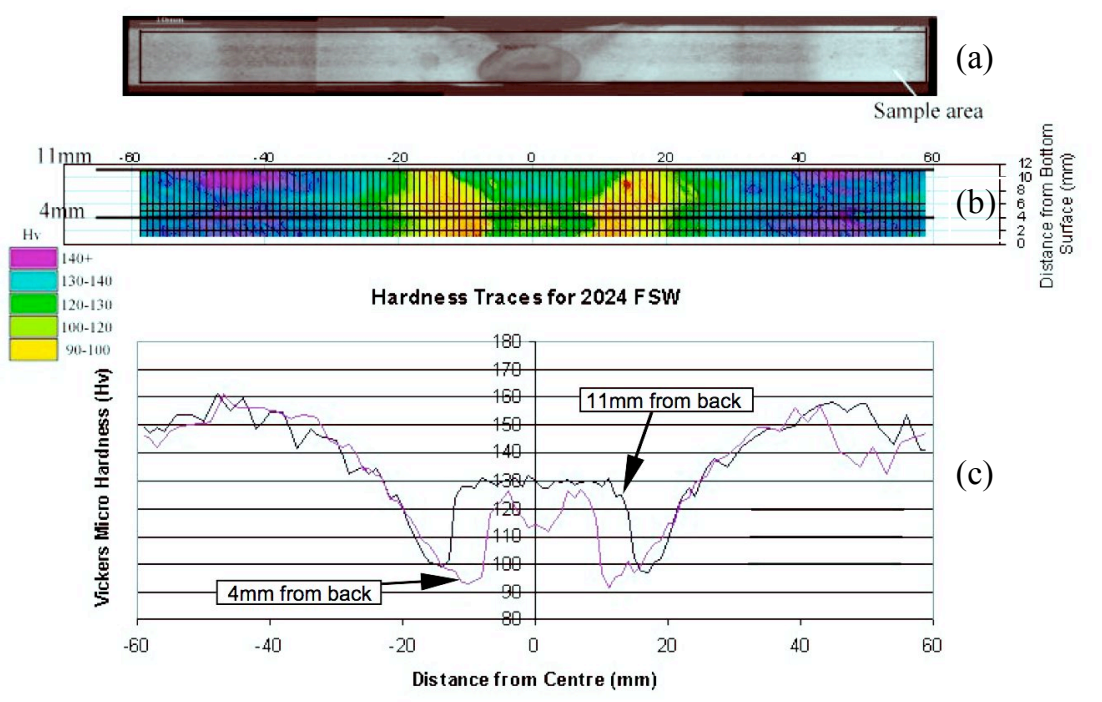

Figure 3 Hardness characteristics for 2024-T351 friction stir welded plate: (a) optical macrograph, (b) hardness map plotted to same scale as (a), and (c) hardness traces at 4 and $11 \mathrm{~mm}$ from parent plate back, corresponding to traces through the centre of the weld nugget and the flow arm region respectively (trace positions are highlighted in (b)).

\section{Mechanical Testing}

Fatigue tests were carried out transverse to the weld line (i.e. perpendicular to the primary expected residual stresses) using four point bend samples of length $300 \mathrm{~mm}$ and with widths of $\sim 40 \mathrm{~mm}$, with an inner roller spacing of $90 \mathrm{~mm}$ (the uniform stress area encompassing the main features of the weld, TMAZ \& HAZ regions). A nominal surface stress range of $170 \mathrm{MPa}$ was applied to the weld top surface in the present experiments, using an R-ratio of 0.1 and a loading frequency of $10 \mathrm{~Hz}$. All specimens were skimmed by $2 \mathrm{~mm}$ relative to the parent plate dimensions (top and bottom surfaces). Fatigue test surfaces were mechanically polished to a $1 / 4$ micron finish and lightly etched in Keller's reagent. Fracture surfaces were examined optically and in the scanning electron microscope (SEM).

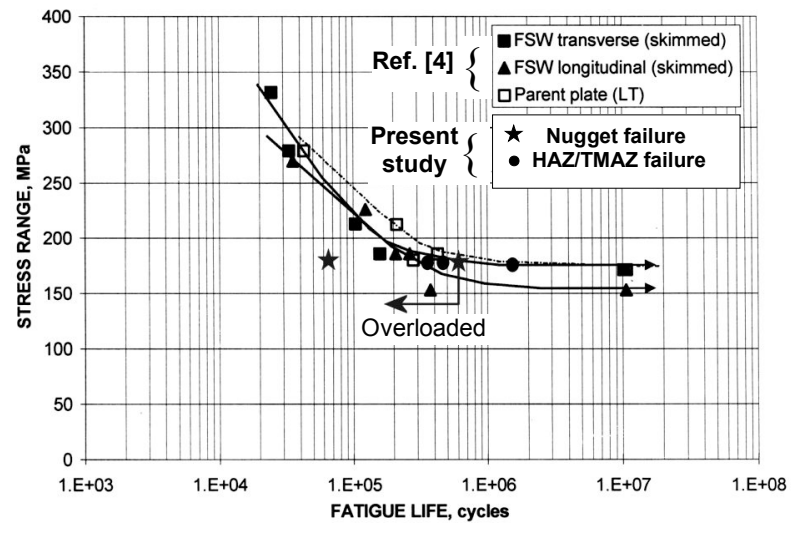

Figure 4 Fatigue life vs. stress range results for present 2024 welds and comparable transverse and longitudinal tests of FSWs in $6 \mathrm{~mm} 2024$ plate (from Bussu and Irving [4]). All data correspond to $\mathrm{R}=0.1$.

\section{Fatigue Results}

Of the five samples that were available for testing, two forms of failure were identified: (i) tests where failure occurred from within the actual weld material (identified as 'nugget failure'), and (ii) tests where failure occurred outside of the actual weld, either in the TMAZ or HAZ. In terms of fatigue life it may be seen from Fig. 4 that for the three specimens where failure occurred outside of the nugget region, fatigue performance was comparable to that found by Bussu and Irving [4] for FSWs in thinner plate and parent material. Two specimens failed over the weld nugget region, 
although accurate fatigue life data is only available for one sample. The fatigue life of this sample was appreciably lower (by a factor of $\sim 10$ ) than the specimens that failed outside the nugget region. The other sample that failed over the weld nugget received a slight overload during testing, hence the overall life is not directly comparable to the other data: the failure mechanism of the sample is however of interest (see below).

\section{Fractography}

Failures Outside the Nugget Region. Fatigue failure in this region was characterised by macroscopically flat crack growth, perpendicular to the applied load. For two of the samples, initiation was located $\sim 15 \mathrm{~mm}$ from the weld centreline (see Fig. 3(c)), on the steep rise in hardness between the HAZ/TMAZ minima and central weld plateau (i.e. not at the minimum point). The other failure outside the nugget region occurred some $30 \mathrm{~mm}$ from the weld centreline, i.e. at a point just within the initial hardness peak of the HAZ. In each case initiation occurred by decohesion of large S-phase particles, with subsequent crack propagation showing essentially classical stage I transgranular failure, giving way to more ductile crack propagation with increasing crack length.

Failure Over the Nugget Region. In both instances of fatigue failure over the nugget, initiation was identified with a distinct macroscopic discontinuity in the material flow pattern at the surface of the sample, as highlighted in Fig. 5 (showing top view of the broken specimen halves). Samples that failed in the HAZ/TMAZ did not exhibit such features. Crack growth across the nugget region was macroscopically more irregular than that seen for failure in the HAZ/TMAZ. Crack growth down through the weld flow arm showed minor deflections from a nominal mode I path (see the top part of Fig 6), however marked deflection was seen when the crack reached the weld nugget, with subsequent failure closely following the "onion ring" banding of the nugget region, see Figs $6 \& 7$. The crack was found to have grown just within the weld nugget, rather than at the nugget/TMAZ interface.

After careful SEM investigation the exact microscopic cause of crack initiation over the weld nugget was not considered clearly identifiable, with no obvious defects being seen (e.g. local porosity or course intermetallic particles). The growth mode was predominantly transgranular with some fine scaled intergranular features (i.e. of the order of the grain size in the flow arm and nugget regions).

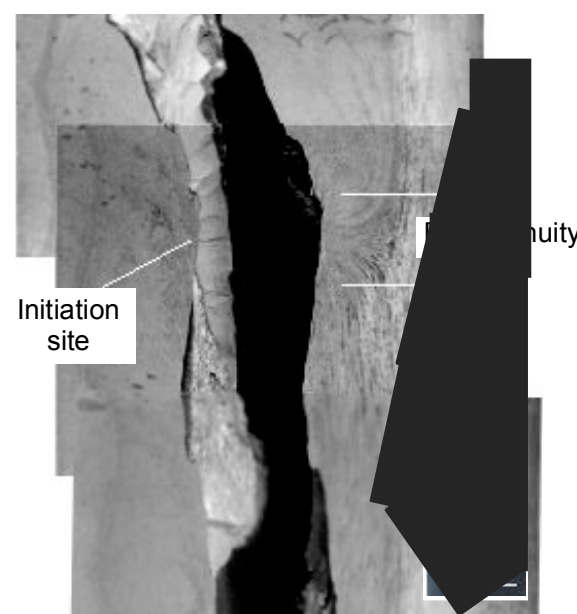

Figure 5 Top surface view of specimen halves for failure over the weld nugget.

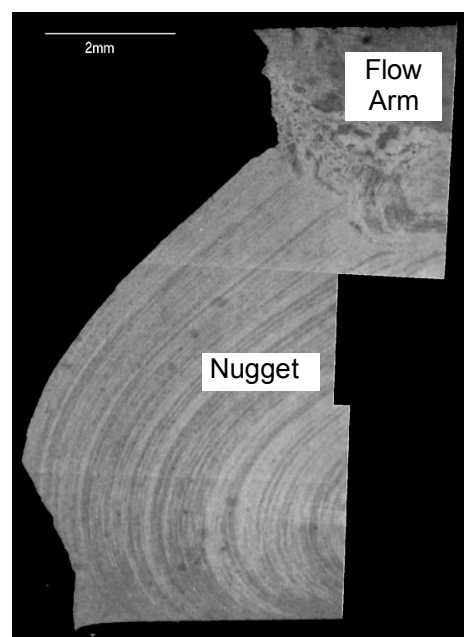

Figure 6 Macrograph showing fatigue crack path down through the weld region

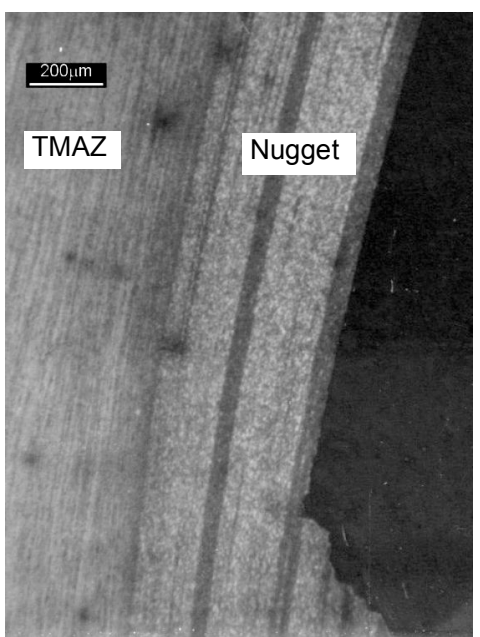

Figure 7 Crack path following the edge of a dark band within the weld nugget. 


\section{Discussion}

In the first instance several microstructural features of the FSW weld nugget/flow arm would appear conducive to good fatigue life performance this region, specifically: (a) the fine grain dimensions and intermetallic particle distribution, (ii) moderate strength levels, and (iii) the very low void/defect content introduced by the present FSW process. As such, the incidence of essentially conventional crack initiation processes in the HAZ/TMAZ, and the good overall fatigue performance of the welds, would appear reasonable. Initiation of cracks at coarse intermetallic particles (when failure occurred in the HAZ/TMAZ region) is of course typical of commercial heat treatable alloys, although it may be presumed that changes in strengthening precipitate features such as shearability and/or the incidence of heterogeneous precipitation at particle/matrix interfaces may influence the effective decohesion strength of the intermetallics at a specific location. The fact that initiation did not occur at the hardness minima shows that local flow strength is not the primary influence on initiation behaviour, as reported in [4].

Whilst the exact microstructural origins of crack initiation within the flow arm were not clear, it is apparent that, in keeping with the crack deflection that occurred at the weld nugget, preferred failure behaviour in FSWs is directly influenced by flow features of the process. In terms of the deflected crack growth at the nugget, Norman et al [6] have identified the onion ring bands in 2024T351 FSWs with abrupt changes in intermetallic particle density, with limited apparent changes in local grain dimensions and preferred crystallographic orientations. Changes in intermetallic distribution at the edge of an individual onion ring band in the present welds are highlighted in Fig. 8. Backscattered electron imaging of crack growth through the bands showed limited interaction with the intermetallic particle distribution, i.e. the particles did not provide the preferential crack path around the nugget bands.

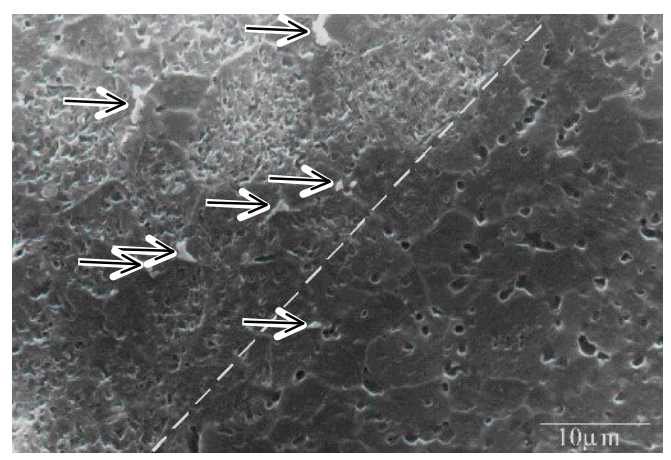

Figure 8 SEM image of the interface between two bands making up the "onion ring" structure within the weld nugget (polished and etched in Keller's reagent): the dashed line highlights the band edge, whilst arrows highlight the location of larger intermetallics.

It is interesting to note the change in intragranular etching characteristics at the band edge in Fig. 8, with the upper-left part of the micrograph showing marked intragranular attack, presumably due to changes in coarse matrix precipitation. Marginal changes in grain dimensions were also noted between the bands (cf. [6]). To further access microstructural variations between the bands in the weld nugget, local hardness measurements are shown in Fig. 9. The figure shows a consistent hardness variation of the order of $10 \mathrm{H}_{\mathrm{v}}$ between the harder light bands and the comparatively soft dark bands. In the first instance the deflected nature of crack growth in the weld nugget may then be a consequence of the mechanical interaction between a crack and layers of hard and soft material, with the results of Suresh et al. [7] for example showing the reduction in crack driving force that may occur as a crack moves from a layer of low yield strength to one of higher strength. Whilst the measured hardness changes are only of the order of $10 \%$ and additional intrinsic influences of microstructural variations between bands (as evidenced by the hardness changes in themselves) cannot be ruled out, the 'sense' of the crack deflections in the present welds is consistent with such a layer hardness effect, with deflection around the nugget occurring at transitions between low to high hardness material (see Fig. 7). 


\section{Conclusions}

1. Two locations have been identified for fatigue crack initiation: within the HAZ/TMAZ and over the weld nugget. Fatigue lives of specimens that fail outside the nugget region were comparable with published results for parent plate and the results of Bussu and Irving [4] for FSWs in thinner 2024-T351 material. Crack initiation over the nugget region would appear to compromise fatigue life.

2. Fatigue failure was influenced by the apparent flow features of the FSW process, with crack initiation within the weld region being related to the presence of macroscopic discontinuities in the flow pattern of the flow arm. Subsequent crack propagation into the weld nugget was seen to follow the associated onion ring band structure. Differences in hardness between the bands have been identified, consistent with a mechanical contribution to crack deflection behaviour.

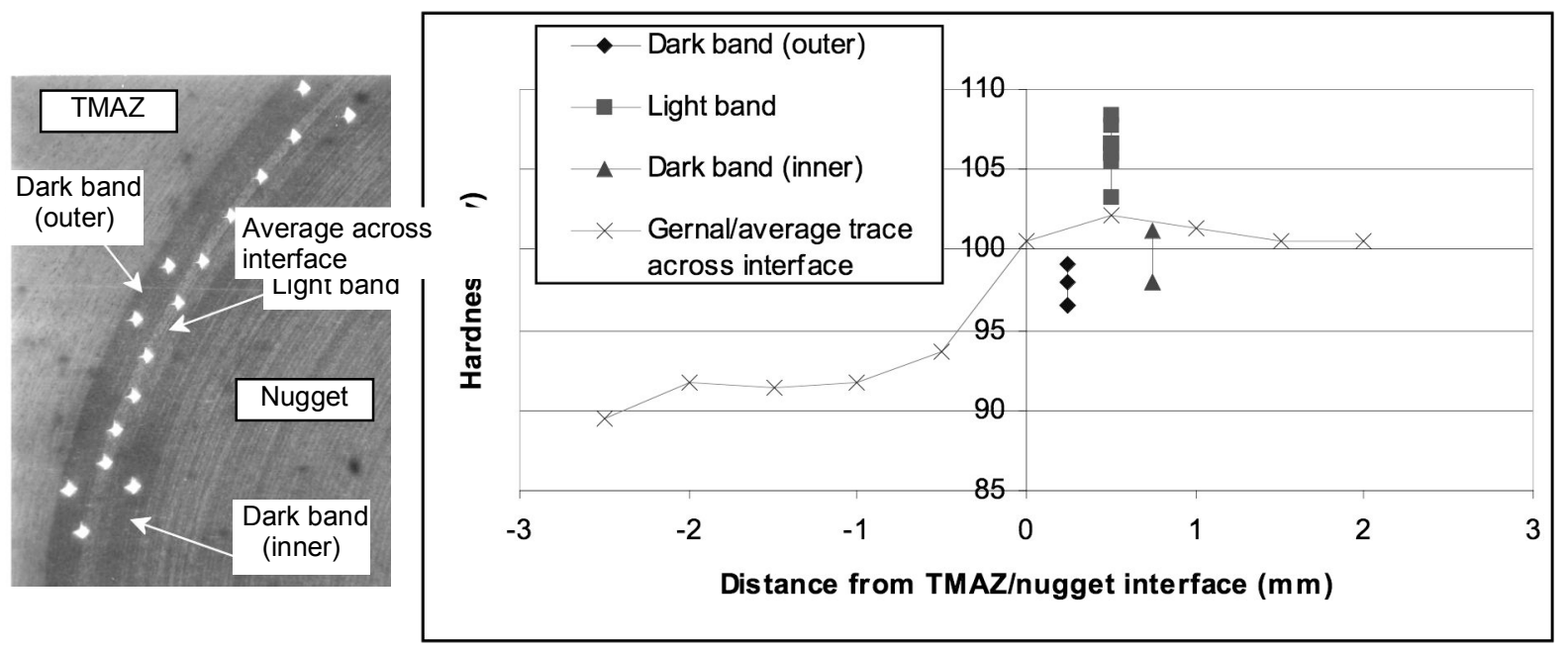

Figure 9 Hardness variations within the nugget onion ring bands.

Acknowledgements: The authors wish to thank Airbus UK for materials supply and financial support, and the EPSRC for financial support.

\section{References:}

[1] R. Pedwell, H. Davies and A. Jefferson, 1999, Proc. $1^{\text {st }}$ Int. Symposium on Friction Stir Welds, Thousand Oaks, California, $14^{\text {th }}-16^{\text {th }}$ June.

[2] L.E. Murr, Y. Li, E.A. Trillo, B.M. Nowak and J.C. McClure, 1999, Alumnum Transactions, Vol.1, pp. 141-154

[3] C.G. Rodes, M.W. Mahoney, W,H, Bingel, R.A. Spurling and C.C Bampton, 1997, Scripta Materialia, Vol. 36, pp. $69-75$

[4] G. Bussu and P.E. Irving, 1999, Proc. ${ }^{\text {st }}$ Int. Symposium on Friction Stir Welds, Thousand Oaks, California, $14^{\text {th }}$ $-16^{\text {th }}$ June.

[5] C.Y. Kung and M.E. Fine, 1979, Metallurgical Transactions A, Vol. 10A, pp. 603-610

[6] A.F. Norman, I. Brough and P.B. Prangnell, 2000, Materials Science Forum, Vols 331-337, pp. 1713-1718.

[7] S. Suresh, Y. Sugimura and E.K Tschegg, 1992, Scripta Metallurgica, Vol. 27, pp. 1189-1194

This document is available on the web at http://www.ttp.net/downloads

\section{爬อ Trans Tech Publications Ltd}

Brandrain 6

Fax: $\quad+41-19221033$

CH-8707 Uetikon-Zuerich

e-mail: ttp@ttp.net

Switzerland

Web: http://www.ttp.net 